\title{
Extramedullary Involvement by Chronic Myelogeneous Leukemia in Five Patients With Unusual Clinicopathologic Features: A Review of the Effectiveness of Tyrosine Kinase Inhibitors
}

\author{
Abha Soni $^{\mathrm{a}}$, Ravikumar Paluri ${ }^{\mathrm{b}}$, Taylor Deal ${ }^{\mathrm{a}}$, Deniz Peker ${ }^{\mathrm{a}, \mathrm{c}}$
}

\begin{abstract}
Chronic myelogeneous leukemia (CML) is associated with BCRABL1 fusion gene leading to an abnormal tyrosine kinase molecule. The accepted first-line treatment is imatinib mesylate (IM). CML uncommonly occurs in the extramedullary sites at initial presentation or relapse. Here we report five adult patients with CML who developed myeloid sarcoma (MS) while on treatment with IM. A retrospective medical chart analysis was performed to identify CML patients with MS who were diagnosed and treated at the University of Alabama at Birmingham. The age ranged between 21 and 36 years (median: 28.5) with a male to female ratio of $4: 1$. All of the patients were diagnosed with CML in chronic phase with initial treatment including IM. The median interval period between the initial diagnosis of CML and MS was 27 months (range 7 - 60 months). The sites of extramedullary involvement included lymph nodes $(\mathrm{n}=2)$, central nervous system $(\mathrm{n}=$ $2)$ and hepatobiliary organs $(n=1)$. All patients were treated with either induction therapy or stem cell transplant (SCT) following the diagnosis of MS. The median survival was 16 months (range 1 - 72 months). The longest survival was observed in a patient who successfully received SCT therapy. IM is frequently used as the first therapeutic choice in new diagnosed CML; however, its penetration and effectiveness in extramedullary tissue is still unclear. The current report also supports the literature with less favorable prognosis of CML in younger individuals.
\end{abstract}

Keywords: CML; Myeloid sarcoma; Tyrosine kinase inhibitors

\section{Introduction}

Chronic myelogeneous leukemia (CML) is a myeloproliferative neoplasm characterized by a reciprocal translocation of

Manuscript accepted for publication April 13, 2016

aDepartment of Pathology, University of Alabama in Birmingham, Birmingham, AL, USA

bepartment of Internal Medicine, Division of Hematology \& Oncology, University of Alabama at Birmingham, Birmingham, AL, USA

${ }^{c}$ Corresponding Author: Deniz Peker, Department of Pathology, University of Alabama at Birmingham, 619 19th Street South, NP 3552, Birmingham, AL 35249-7331, USA. Email: dpeker@uab.edu

doi: http://dx.doi.org/10.14740/jocmr2566w chromosomes 9 and 22, which leads to a fused BCR/ABL gene product and induces the production of an abnormal tyrosine kinase molecule [1]. CML accounts for approximately $14 \%$ of leukemias worldwide with an incidence of 1 - 2 per 100,000 persons $[1,2]$. CML is historically considered as a disease of older adults with a reported median age of 67 [3]. However, recent studies and trials revealed a growing number of CML patients younger in age, potentially exhibiting a more aggressive disease [4]. CML is a primary disease of bone marrow and is classically divided into three phases (based on blast count and other parameters): chronic, accelerated, and blast crisis. Most patients initially present in chronic phase with elevated white blood cell (WBC) counts consisting mostly of neutrophils and myelocytes accompanied by basophilia and a normal to increased number of platelets in the peripheral blood. The bone marrow is hypercellular with granulocytic predominance, increased number of, often morphologically abnormal, megakaryocytes with or without reticulin fibrosis, and $<5 \%$ blasts. Constitutional symptoms such as weight loss, night sweats, and fatigue can also be appreciated $[1,5,6]$.

Myeloid sarcoma (MS) is a rare form of myelogenous leukemias and refers to involvement of extramedullary (EM) sites including bone, skin, or lymph node [7,8] and confers a worse prognosis [9]. Leukemia cutis (LC) is a specific form of MS characterized by leukemic cells specifically infiltrating the dermis $[1,10]$. Literature suggests that increased risk of EM involvement occurs through alteration of tissue-homing pathways [7].

EM manifestation of CML is very rare. It is reported in large cohort studies and also sporadically in patients diagnosed with the accelerated or blast crisis phase of CML $[8,11,12]$. Here, we report a single institution experience on salient clinicopathological, phenotypic, and molecular characteristics of MS in a series of adult CML cases.

\section{Case Reports}

\section{Patients and clinical characteristics}

Five cases of CML with blast crisis in the form of EM disease were retrieved from the files of the Department of Pathology at the University of Alabama at Birmingham (UAB). Clinical and laboratory information and follow-up data were obtained from 
Table 1. Clinicopathological and Molecular Characteristics of CML Patients With Extramedullary Disease

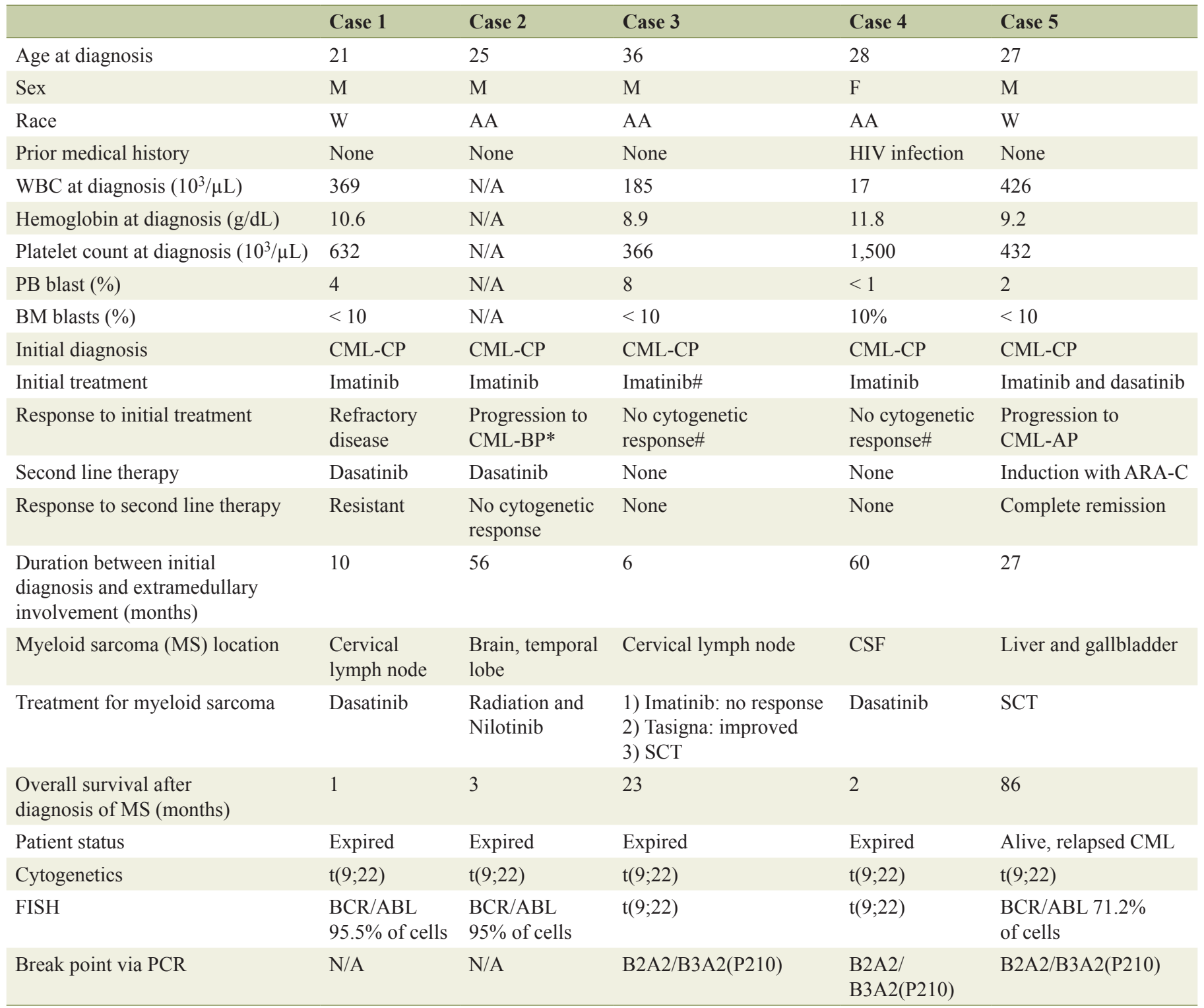

*Patient 2 was treated with ARA-C and Gleevec $(800 \mathrm{mg})$ for CML-BP with morphologic response and no cytogenetic response. \#Patients 3 and 4 had hematologic response and partial cytogenetic response to initial treatment with imatinib (400 mg daily); however, disease progressed due to patient incompliance.

medical chart review. Clinicopathological parameters included age, gender, primary diagnosis, location of EM involvement, tumor cell phenotype, status of bone marrow involvement at time of diagnosis, prior treatment, conventional cytogenetic and fluorescence in situ hybridization (FISH) analysis results, and when available other molecular testing results. Relevant patient data are summarized in Table 1. The study was approved by the Institutional Review Board of UAB.

\section{Case 1}

A 21-year-old African American male presented with abdomi- nal pain in November 2005. Initial radiography showed massive splenomegaly without masses or lesion anywhere else. Blood analyses revealed moderate normocytic normochromic anemia, marked leukocytosis with WBC count of 369,300/ $\mu \mathrm{L}$, and an increase platelet count of $632,000 / \mu \mathrm{L}$. The initial bone marrow biopsy revealed CML in chronic phase (CMLCP) with $\mathrm{t}(9 ; 22)(\mathrm{q} 34 ; \mathrm{q} 11.2)$ via conventional karyotyping and BCR-ABL fusion transcripts in $95.5 \%$ of cells analyzed by FISH. The patient was initiated on imatinib (400 mg daily). The WBC count reduced to $23,000 / \mu \mathrm{L}$ in the following 2 months without cytogenetic remission. In the next few months of the clinical course, the WBC count increased up to $90,000 / \mu \mathrm{L}$ with persistent CML-CP which led to an increase in imatinib dose 
(600 mg daily). However, no clinical or cytogenetic remission was achieved. Furthermore, the patient's abdominal pain worsened and he developed significant ascites. Cytological examination of the paracentesis fluid revealed increased early hematopoietic precursors. The corresponding flow cytometry demonstrated 10\% blasts expressing CD34, CD33, and CD13 without CD117. Subsequently, he developed cervical lymphadenopathy (LAP), confirmed as MS on biopsy. A follow-up bone marrow showed CML-accelerated phase (CML-AP) with $14 \%$ blasts. The disease was considered imatinib-resistant and he was started on dasatinib (140 mg daily) with improvement of WBC. However, his bone marrow biopsy showed persistent CML-CP with BCR-ABL1 in $91 \%$ of cells by FISH. Despite the hematologic response, he subsequently developed multiregional LAP with MS. He was diagnosed with CML-blasts crisis and proceeded to induction chemotherapy. The LAP improved and at day 14 the bone marrow showed no evidence of disease. However, within a month, the patient expired due to treatment-related complications.

\section{Case 2}

A 25-year-old African American male was diagnosed with CML-CP at an outside institution in November 2001. He was initially observed and then received Gleevec, which controlled his disease. In January 2005, he transformed to blastic phase and received induction therapy with ARA-C, followed by Gleevec ( $800 \mathrm{mg}$ daily), without cytogenetic remission. In May 2005, he presented to UAB with severe fatigue and weakness in addition to weight loss. He had a WBC count of $138,000 / \mu \mathrm{L}$ with $28 \%$ blasts. A follow-up bone marrow biopsy revealed AML with $90 \%$ myeloblasts. Dasatinib treatment as a part of clinical trial was initiated and he was also put on hydrea and allopurinol in order to decrease the cell count. The disease was well controlled over the course of 12 months. In May 2006, he developed blast crisis for which he received two cycles of MEC chemotherapy. He was then enrolled in the SKI-606 (bosutinib) study and had a remarkable response. However, while on this study drug, he developed severe headaches and imaging studies revealed a large temporal mass. This area was biopsied and diagnosed as MS. The patient received radiation therapy with complete resolution of the mass and had no hematologic disease. In the following next months, the patient had relapsed central nervous system (CNS) disease, diagnosed in cerebrospinal fluid (CSF), necessitating more radiation. He was then included in a study with nilotinib for tyrosine kinase inhibitor resistant disease and failed to show a response. Throughout his clinical course, the complications of treatment included MRSA bacteremia, pericardial tamponade, and aspergillus pneumonia. Additionally, he underwent splenectomy for chronic thrombocytopenia. The patient expired in the next 2 months.

\section{Case 3}

A 36-year-old African American male presented with cervical LAP, massive splenomegaly, and sore throat in March 2009.
Blood work revealed a WBC count of $185,000 / \mu \mathrm{L}$, hemoglobin of $8.9 \mathrm{~g} / \mathrm{dL}$, hematocrit of $26 \%$, and a platelet count of $366,000 / \mu \mathrm{L}$. There were $8 \%$ myeloblasts by flow cytometry expressing CD34, CD117, CD30, CD33, and HLA-DR. Cytogenetics showed a male karyotype with 9:22 translocation in all six metaphases. BCR/ABL by PCR was positive in $36.7 \%$ of bone marrow cells. The core biopsy revealed a maturing myeloid expansion with hypolobate megakaryocytes. He was diagnosed with CML-CP and initiated on imatinib $(200 \mathrm{mg}$ daily). Initially he tolerated it well, but by the following week, his WBC count had decreased to $146,000 / \mu \mathrm{L}$. In early May, his WBC count was down to $9,200 / \mu \mathrm{L}$, hemoglobin was 11 $\mathrm{g} / \mathrm{dL}$, and hematocrit was 33\%. In July 2009, although he had achieved a hematologic remission, with a WBC count of $8,860 / \mu \mathrm{L}$, he continued to have cervical and axillary LAP. Quantitative BCR/ABL was high, showing that he was only in a partial cytogenetic remission. Over the next few months, he presented to the emergency department with low-grade fevers, joint pain, and constipation. He reported that he temporarily discontinued imatinib, but resumed as symptoms recurred. In October, a right cervical neck node biopsy was obtained. The histology report showed partial replacement of the lymph node by immature myeloid cells. Flow cytometry of the node showed a myeloid population with $4-4.5 \%$ myeloblasts expressing CD30, 33, 34, 38, 45, CD117, 123, and HLA-DR. After 7 months of starting imatinib, his WBC count was down to $2,700 / \mu \mathrm{L}$, hemoglobin was $8.76 \mathrm{~g} / \mathrm{dL}$, hematocrit was $27.6 \%$, and platelet count was $93,000 / \mu \mathrm{L}$. In September, his WBC count had increased to $202,000 / \mu \mathrm{L}$, he was febrile and his peripheral blood showed $30 \%$ basophils and $4 \%$ myeloblasts. His bone marrow was hypercellular at $50 \%$ with $15 \%$ blasts. A quantitative BCR/ABL PCR done in November was found to have increased from initial presentation.

A mutational analysis showed an ABL kinase mutation of G250E in $8.25 \%$ of cells. There was no evidence of T315I mutation. He was diagnosed as an accelerated phase CML and switched to tasigna. He remained on tasigna to maintain his chronic phase CML before transplant. He received a stem cell transplant (SCT) in February 2011. Post-transplant, he developed neutropenic sepsis requiring IV broad-spectrum antibiotics. In October 2011, he presented with skin, oral, gastrointestinal, and hepatic graft versus host disease. He subsequently became hemodynamically unstable with venous bleed from the anterior edge of liver, massive hemorrhagic ascites, progressive liver failure, DIC, lactic acidosis, cyclosporine-induced renal insufficiency, and acute respiratory distress syndrome. He succumbed to multiorgan failure and expired in November 2011.

\section{Case 4}

A 28-year-old African American female with a history of HIV and seizure disorder presented in 2006 with an elevated platelet count $\left(1,500 \times 10^{3} / \mu \mathrm{L}\right)$. A bone marrow aspirate revealed abundance of myeloid cell lineage, with less than $10 \%$ blasts, followed by megakaryocytic, and finally the erythroid lineages. The bone marrow biopsy showed $90-95 \%$ cellularity. She was placed on imatinib (400 mg twice daily), to which she 
showed a hematologic response, but she was non-compliant due to gastrointestinal symptoms. At the same time, she was also diagnosed with HIV/AIDS and started on highly active antiretroviral therapy (HAART). In March 2011, she presented with bilateral lower extremity weakness, which was thought to be due to spinal cord enlargement/infiltration on MRI. Her CSF showed 22 cells/dL, mostly small to intermediate lymphocytes, an occasional monocyte, and two neutrophils. Although no blasts were seen in the CSF, her presentation was most consistent with EM manifestation of CML. She also had generalized LAP, the most prominent being the right anterior cervical lymph node measuring $4 \times 3 \mathrm{~cm}$. The LAP was probably related to CML or infectious, immune etiology. No lymph node biopsy was obtained. Her spleen was palpable $7-8 \mathrm{~cm}$ below the left costal margin. At that time, her hemoglobin was $9.6 \mathrm{~g} / \mathrm{dL}$, WBC count was $133,000 / \mu \mathrm{L}$, neutrophils was $49 \%$, lymphocytes was $3 \%$, blasts was $4 \%$, monocytes was $21 \%$, and platelet count was $572,000 / \mu \mathrm{L}$. In April 2011, she was treated with dasatinib (140 mg daily), but she developed febrile neutropenia with an ANC of 352. She was started on vancomycin, ceftazidine, and fluconazole for oral thrush. She also underwent incision and drainage of right groin abscess. Dasatinib was temporarily withheld. Her ANC improved to 1,260. She was also maintained on antiretroviral therapy during the hospital stay and was counseled to follow-up with the HIV clinic. The goal was to restart anti-CML therapy, but after May 2011, she was not able to tolerate treatment due to deteriorating medical condition. She was readmitted to the hospital with exacerbation of symptoms in July 2011, with anemia, acute renal injury, uncontrolled type 1 diabetes mellitus, dehydration lactic acidosis, and worsening respiratory failure. She received palliative care in the hospital and died shortly after.

\section{Case 5}

A 28-year-old otherwise healthy white male presented with remarkably high WBC count of $426 \times 10^{3} / \mu \mathrm{L}$ in February 2006 . He had moderate anemia and normal platelet count. A peripheral blood and bone marrow biopsy examination revealed features of CML in chronic phase. The diagnosis was confirmed with molecular testing. The patient was started on imatinib (400 mg) and WBC count was reduced. However, complete clinical or cytogenetic remission was not achieved, despite the increased dose at $600 \mathrm{mg}$. The treatment was changed to dasatinib at $140 \mathrm{mg}$ without significance cytologic response. The patient was followed and evaluated for an SCT. When on dasatinib therapy, he progressed to blast phase in 2 years and was treated with high-dose ARA-C induction therapy. A complete remission was achieved for AML without cytogenetic remission for CML. He was followed on dasatinib until April of 2008. At this time, he developed multiple lesions in the liver and gallbladder. The liver lesions were microscopically examined and a diagnosis of MS was rendered based on the phenotype $\left(\mathrm{CD} 34^{+}, \mathrm{CD} 117^{+}\right.$, and $\left.\mathrm{CD} 33^{+}\right)$. The patient immediately received allogeneic SCT from an unrelated donor and remained disease free until July of 2014, when he presented with relapsed CML-CP.

This patient is still alive without current evidence of dis- ease progression.

\section{Discussion}

EM blast crisis can occur in CML with a prevalence reported to be around $15 \%$ and most commonly involves bone, lymph nodes, skin, soft tissue, and CNS [13]. The incidence of EM blast crisis in the newly diagnosed patients is extremely rare. EM blast crisis, after a few months, is almost always followed by hematological blast crisis, therefore considered as the earliest sign, or pathognomonic for, blast crisis in the bone marrow $[13,14]$. Blast crisis remains a challenge in the management of CML, especially in those with EM presentation. The introduction of BCR-ABL tyrosine kinase inhibitors has changed the clinical outlook of CML with remarkable improvement in survival in chronic-phase CML with a complete cytogenetic response in $60-70 \%$ of patients [15]. However, the number of effective treatment options is limited in advanced-phases of CML. The median survival after diagnosis of blast crisis or EM relapse currently ranges from 7 to 11 months compared to 3 - 4 months in the pre-imatinib era, which illustrates a less than desirable improvement and an unmet need [16]. Similarly, the median survival in our case series was 16 months. The survival was remarkably long in case 5 who successfully received SCT and remained disease free for more than 70 months.

Available therapeutic options for newly diagnosed EM CML, tyrosine kinase inhibitor-naive patients include imatin$\mathrm{ib}$, dasatinib, or nilotinib [17]. Better clinical outcomes were reported for the few patients who return to chronic phase with treatment and are successfully transplanted [18]. Even in the imatinib era, the best outcomes continue to be observed for patients who undergo allogeneic SCT. According to the German CML Study Group, the 3-year overall survival of 28 imatinibpretreated patients transplanted in advanced phase $(25 / 28$ in blast crisis) was 59\% [18]. Therefore, guidelines recommend considering allogeneic SCT after a reasonable attempt has been made with a suitable TKI-selected therapy, according to the mutation profile, to return the patient to chronic phase and then do SCT. If TKIs alone are not sufficient, acute leukemia-type induction therapy could be pursued. Two out of five patients in our series received SCT, with one patient (case 5) achieving complete clinical and cytogenetic remission for 74 months until a diagnosis of relapsed CML. The other patients, unfortunately, developed multiple complications following SCT and expired shortly after.

Post-TKI EM relapse has been reported previously. Isolated EM blastic crises have been reported in the CNS after being in hematological remission by IM [19]. In our series, patients reported in cases 2 and 4 had CNS involvement. It can be speculated that the disease progression in the CNS was most likely due to failure to achieve adequate level of imatinib in the CNS, rather than systemic resistance to imatinib. The CNS has relatively poor penetrance for imatinib and could serve as a sanctuary site for leukemic cells [20]. Disease progression from these sanctuary sites can occur in the long term. Inadequate IM levels in tissues might be associated with treatment failure in these patients, leading to EM CML progression. A 
second generation TK inhibitor, i.e. dasatinib, has been suggested to be a useful therapeutic strategy for these patients, as dasatinib can penetrate EM tissues including CNS relatively well [21]. However, our patient (case 2) progressed to EM disease, despite therapy with dasatinib.

Resistance to IM can also result from individual genetic variations in addition to drug-drug and drug-diet interactions that affect imatinib metabolism by altering cytochrome P450 $3 \mathrm{~A} 4$ activity [22]. IM efficacy may be influenced both locally and systemically by changes in the intracellular distribution of unbound drug [23]. The possible role of protein binding to $\alpha 1$ acid glycoprotein, which affects the serum level of imatinib, has been reported [24]. The occurrence of abnormal $\mathrm{Ph}^{+}$clones as well as clonal evolution of $\mathrm{Ph}^{+}$cells has also been reported in CML patients under imatinib treatment. It has been suggested that abnormal clones may arise from a $\mathrm{Ph}^{-}$stem cell in a pre-leukemic stage or may represent the clonal selection by imatinib in $\mathrm{Ph}^{-}$or in $\mathrm{Ph}^{+}$stem cells with accumulated genetic lesions, occurring also in EM tissues [25].

EM involvement of CML is difficult to control. Although many patients will have an initial response to TKI therapy, such responses are often short-lived. Therefore, a reasonable plan is an attempt to return the patient to a second chronic phase using second generation TKIs with suitable candidates subsequently undergoing allogeneic SCT. It is possible that with longer follow-up, more patients with CML achieving molecular remission with imatinib will relapse at unusual sites. Such EM manifestations may not be detected early by regular molecular monitoring of the blood. Therefore, physicians utilizing imatinib for the treatment of CML should remain vigilant to unusual signs and symptoms. Future research should focus on further investigating the role of second generation tyrosine kinase inhibitors and conducting controlled prospective studies that improve treatment strategies for these complicated CML cases exhibiting EM relapse.

\section{Competing Interests}

There are no financial or non-financial competing interests to declare in relation to this manuscript.

\section{Author Contributions}

AS, RP and DP analyzed the data and wrote the manuscript. TD and DP collected the data.

\section{References}

1. Swerdlow SH, Cancer IAfRo, Organization WH. WHO classification of tumours of haematopoietic and lymphoid tissues. International Agency for Research on Cancer. 2008.

2. Besa EC. Is minimal residual disease in the peripheralblood stem-cell transplantation of chronic myelogenous leukemia important? J Clin Oncol. 1997;15(9):3166-
3167.

3. Siegel R, Naishadham D, Jemal A. Cancer statistics, 2013. CA Cancer J Clin. 2013;63(1):11-30.

4. Kalmanti L, Saussele S, Lauseker M, Proetel U, Muller MC, Hanfstein B, Schreiber A, et al. Younger patients with chronic myeloid leukemia do well in spite of poor prognostic indicators: results from the randomized CML study IV. Ann Hematol. 2014;93(1):71-80.

5. Tefferi A. Classification, diagnosis and management of myeloproliferative disorders in the JAK2V617F era. Hematology Am Soc Hematol Educ Program. 2006:240245.

6. Jabbour E, Kantarjian H. Chronic myeloid leukemia: 2014 update on diagnosis, monitoring, and management. Am J Hematol. 2014;89(5):547-556.

7. Bakst RL, Tallman MS, Douer D, Yahalom J. How I treat extramedullary acute myeloid leukemia. Blood. 2011;118(14):3785-3793.

8. Pileri SA, Ascani S, Cox MC, Campidelli C, Bacci F, Piccioli M, Piccaluga PP, et al. Myeloid sarcoma: clinicopathologic, phenotypic and cytogenetic analysis of 92 adult patients. Leukemia. 2007;21(2):340-350.

9. Campidelli C, Agostinelli C, Stitson R, Pileri SA. Myeloid sarcoma: extramedullary manifestation of myeloid disorders. Am J Clin Pathol. 2009;132(3):426-437.

10. Cho-Vega JH, Medeiros LJ, Prieto VG, Vega F. Leukemia cutis. Am J Clin Pathol. 2008;129(1):130-142.

11. Specchia G, Palumbo G, Pastore D, Mininni D, Mestice A, Liso V. Extramedullary blast crisis in chronic myeloid leukemia. Leuk Res. 1996;20(11-12):905-908.

12. Agis H, Weltermann A, Fonatsch C, Haas O, Mitterbauer G, Mullauer L, Schreiber S, et al. A comparative study on demographic, hematological, and cytogenetic findings and prognosis in acute myeloid leukemia with and without leukemia cutis. Ann Hematol. 2002;81(2):90-95.

13. Inverardi $\mathrm{D}$, Lazzarino $\mathrm{M}$, Morra $\mathrm{E}$, Bernasconi $\mathrm{P}$, Merante S, Canevari A, Pagnucco G, et al. Extramedullary disease in $\mathrm{Ph}^{\prime}$-positive chronic myelogenous leukemia: frequency, clinical features and prognostic significance. Haematologica. 1990;75(2):146-148.

14. Levy RA, Mardones MA, Burch MM, Krause JR. Myeloid sarcoma as the presenting symptom of chronic myelogenous leukemia blast crisis. Proc (Bayl Univ Med Cent). 2014;27(3):246-249.

15. Perrotti D, Jamieson C, Goldman J, Skorski T. Chronic myeloid leukemia: mechanisms of blastic transformation. J Clin Invest. 2010;120(7):2254-2264.

16. Hehlmann R, Saussele S. Treatment of chronic myeloid leukemia in blast crisis. Haematologica. 2008;93(12):1765-1769.

17. Hehlmann R. How I treat CML blast crisis. Blood. 2012;120(4):737-747.

18. Saussele S, Lauseker M, Gratwohl A, Beelen DW, Bunjes D, Schwerdtfeger R, Kolb HJ, et al. Allogeneic hematopoietic stem cell transplantation (allo SCT) for chronic myeloid leukemia in the imatinib era: evaluation of its impact within a subgroup of the randomized German CML Study IV. Blood. 2010;115(10):1880-1885.

19. Breccia M, Santopietro M, Cannella L, Federico V, Log- 
lisci G, Serrao A, Petrucci L, et al. Isolated central nervous system relapse after nine years of complete molecular remission in a lymphoid blast crisis of chronic myeloid leukemia treated with imatinib. Leuk Res. 2011;35(6):e9192.

20. Isobe Y, Sugimoto K, Masuda A, Hamano Y, Oshimi K. Central nervous system is a sanctuary site for chronic myelogenous leukaemia treated with imatinib mesylate. Intern Med J. 2009;39(6):408-411.

21. Alimena G, Breccia M, Latagliata R, Grammatico S, Matturro A, Capria S, De Propris MS, et al. Dasatinib in the management of lymphoid blast crisis of Philadelphia-positive chronic myeloid leukemia with multiple extra-medullary and intracranial localizations. Leuk Res. 2009;33(8):e134-136.

22. Picard S, Titier K, Etienne G, Teilhet E, Ducint D, Bernard MA, Lassalle R, et al. Trough imatinib plasma lev- els are associated with both cytogenetic and molecular responses to standard-dose imatinib in chronic myeloid leukemia. Blood. 2007;109(8):3496-3499.

23. Streit F, Binder L, Hafke A, Brandhorst G, Braulke F, Haase D, Armbrust T, et al. Use of total and unbound imatinib and metabolite LC-MS/MS assay to understand individual responses in CML and GIST patients. Ther Drug Monit. 2011;33(5):632-643.

24. Gambacorti-Passerini C, Zucchetti M, Russo D, Frapolli R, Verga M, Bungaro S, Tornaghi L, et al. Alpha1 acid glycoprotein binds to imatinib (STI571) and substantially alters its pharmacokinetics in chronic myeloid leukemia patients. Clin Cancer Res. 2003;9(2):625-632.

25. Kantarjian HM, O'Brien S, Cortes JE, Giralt SA, Rios MB, Shan J, Giles FJ, et al. Imatinib mesylate therapy for relapse after allogeneic stem cell transplantation for chronic myelogenous leukemia. Blood. 2002;100(5):1590-1595. 